\title{
Estimation of convective heat losses from conical cavity receiver of solar parabolic dish collector under wind conditions and receiver orientations
}

\author{
Abhinav Rajan, and K. S. Reddy* \\ Heat Transfer and Thermal Power Laboratory, Department of Mechanical Engineering, Indian \\ Institute of Technology Madras, Chennai 600036, India
}

\begin{abstract}
The parabolic dish collector is one of the recognized concentrated solar power systems based on point focusing, which provides high-temperature heat, high concentration ratio, and low heat loss. This system consists of a parabolic reflector and a cavity receiver situated in the focus line. In this work, the conical cavity receiver with an aperture diameter of $0.5 \mathrm{~m}$ is considered for a $100 \mathrm{~m}^{2}$ parabolic reflector having a focal to diameter ratio of 0.48 . Due to the complexity of flow and temperature profile, the estimation of convective heat loss is a difficult task in a cavity receiver. More heat losses are associated with high temperature obtained in the cavity receiver of the parabolic dish collector. Due to diverse wind effect, the convective heat losses ramp up, which significantly influences the thermal performance of the concentrating power system. The present work aims to investigate the heat losses due to convection from the conical cavity receiver. The numerical investigation was performed using ANSYS Fluent 20R1 to calculate the convective heat losses from the conical cavity receiver of varying diameter to height ratio for varying wind speed, receiver orientation in head-on, and back-on wind flow directions. The considered influential parameters are varying from 0.5 to 1.5 for diameter to height ratio $(\mathrm{d} / \mathrm{h}), 0^{\circ}$ to $90^{\circ}$ for receiver orientation $(\gamma), 0$ to $10 \mathrm{~m} / \mathrm{s}$ for wind speed $(\mathrm{V})$. The heat losses are highest at $60^{\circ}$ and $75^{\circ}$ receiver orientation for $\mathrm{d} / \mathrm{h}=0.5$ and $\mathrm{d} / \mathrm{h}=1-1.5$, respectively, at high wind speed in head-on condition, whereas in back-on wind condition, $30^{\circ}$ receiver orientation has more heat losses among all the $\mathrm{d} / \mathrm{h}$ values at high wind speed. The heat loss at $90^{\circ}$ receiver orientation is low for $4-10 \mathrm{~m} / \mathrm{s}$. The trends of heat loss curve at receiver orientations for given wind conditions are similar for velocity more than $2 \mathrm{~m} / \mathrm{s}$. The result reveals that the considered influential parameters have a remarkable effect on convective heat losses from the cavity receiver.
\end{abstract}

\footnotetext{
${ }^{*}$ Corresponding author: ksreddy@iitm.ac.in
} 


\section{Introduction}

The parabolic dish collector is one of the concentrated solar power systems used for hightemperature industrial applications by absorbing the incoming solar energy under varying weather conditions [1]. The parabolic dish collector is a point focus system that consists of a parabolic reflector that reflects the incoming irradiation at the focal point and a receiver that absorbs the concentrated solar energy. The primary purpose of the receiver is to convert absorbed concentrated energy into useful energy with minimal heat and optical losses. The receiver is located in an open ambiance, the wind causes the heat losses from the receiver, and more heat loss has a remarkable influence on the performance of the system. In a practical situation, it is mandatory to estimate the heat loss and improve the performance. The knowledge of heat loss is one of the major concerns in designing an efficient receiver for a parabolic dish solar thermal power system. Generally, the receiver is subjected to hightemperature application in the open atmosphere, all modes of heat transfer are involved. The heat loss through conduction and radiation can be estimated by analytical methods $[2,3]$, while estimating the convective heat loss, as a major part, is quite complex due to the complexity in flow and temperature field nearby the receiver. For the purpose of estimating convective heat loss, many numerical and experimental investigations are performed by researchers in various conditions and for the receiver of different shapes and sizes [4-8]. As per Clausing [9], the volume inside the receiver is divided into two regions, the convective and stagnation region. The heat loss mostly occurs in the convective region and reduces with the reduction of this zone. Both convective and stagnation regions are mainly influenced by the receiver orientation and geometry of the cavity receiver. As orientation changes from $0^{\circ}$ to $90^{\circ}$, the stagnation region starts increasing and reaches the maximum with vanishing the convective region. This is justified by many investigations [10-14]. When the receiver has downward-facing, upswings of hot air occur and envelop the whole cavity with temperature equals to wall temperature and temperature gradient creates a sturdy layer. Though, the geometry of cavity and wall temperature control the flow pattern and regions. By lowering the aperture to surface area ratio, the convective heat loss can be decreased at a maintained surface temperature.

From comprehensive attempts on literature, the geometry configuration, wind speed, and wind condition are the factors which influence the convective loss and consequently the performance of parabolic dish collectors. The analysis performed in this field is limited, and heat loss owing to forced convection is not generic as it is altered by different parameters such as cavity geometrical dimensions, operating ambiances, etc. Therefore, the numerical investigation is carried out in this present work to inspect the effect of wind speeds, wind conditions, receiver orientations, and receiver configuration on convective heat loss from conical cavity receiver of parabolic dish collector. This model can be assumed to be helpful in the estimation of convective heat loss from cavity receivers for process heat applications of medium and high temperatures. The potential application of this receiver can be considered in the Stirling engine to generate electricity from available heat energy.

\section{Description of system}

A conical cavity receiver of an aperture diameter of $0.5 \mathrm{~m}$ has been considered for a largeaperture solar parabolic dish, $100 \mathrm{~m}^{2}$, with a focal to diameter ratio of 0.48 . Fig. 1 shows the conical cavity receiver with orientation details. To lessen the heat loss from the receiver, ceramic insulation is often used to cover the cavity. The parabolic dish collector is one of the efficient concentrated solar systems which reflects the incoming solar radiation to the receiver after hitting the parabolic dish. The receiver absorbs the incoming radiation and converts it into useful work with the help of working fluid moving through the receiver tube 
with the modest heat loss to the neighboring environment. The air is considered as the surrounding medium. The wind speed and direction can be counted as a major concern in more heat loss, which reduces parabolic dish collector performance. The current work considers various influential parameters, such as wind speeds, wind directions, receiver orientations, and geometrical configurations, to observe the effect on heat loss, particularly convective heat loss. The numerical investigation is performed to evaluate heat loss due to convection with the variation in influential parameters such as receiver orientation $(\gamma)$ from $0^{\circ}$ to $90^{\circ}$, wind speed (V) from 0 to $10 \mathrm{~m} / \mathrm{s}$. and diameter to height ratio $(\mathrm{d} / \mathrm{h})$ from 0.5 to 1.5 . The head-on and back-on are considered as wind direction $(\phi)$. The depth, rim angle, and focal length of the parabolic dish are $1.47 \mathrm{~m}, 55^{\circ}$, and $5.42 \mathrm{~m}$, respectively. The effect of the dish may be neglected in back-on due to high rim, focal length, and low curvature, which may not deflect wind towards the receiver[6]. Similarly, in head-on, the dish effect will be marginal due to large elevation [6].

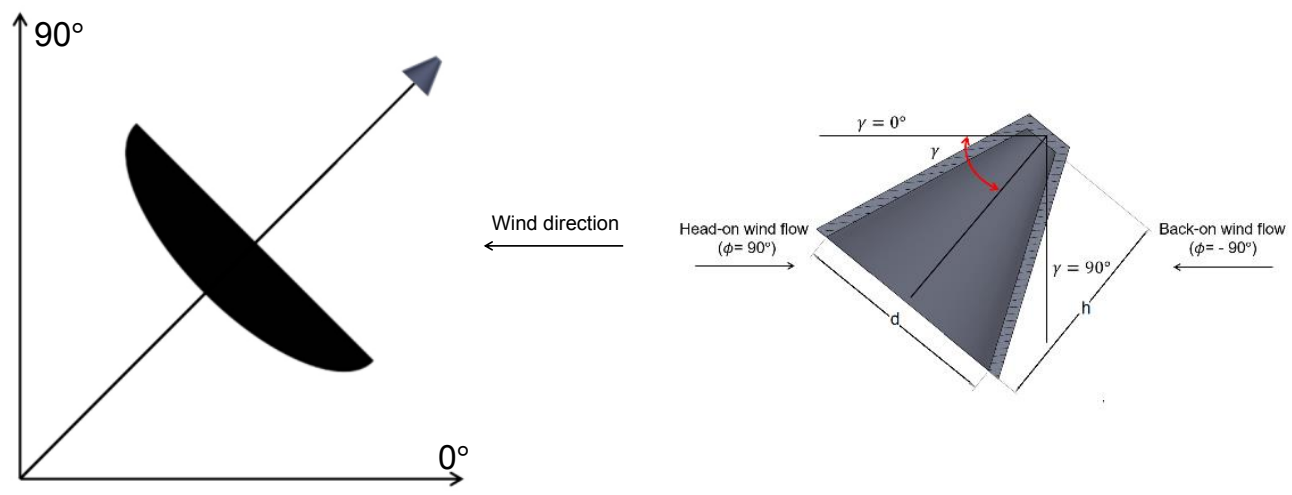

Fig. 1. Definition of receiver orientation $(\gamma)$.

\section{Numerical modeling}

The wind flow in the open ambiance is observed as turbulent. The two-equation model, k- $\omega$ SST (Shear stress transport), is the best suitable turbulence model among the available for flow past a cavity receiver [6]. The governing equations are given as follow:

Continuity equation:

$$
\frac{\partial\left(\rho_{f} u\right)}{\partial x}+\frac{\partial\left(\rho_{f} v\right)}{\partial y}=0
$$

$\mathrm{X}$-momentum equation:

$$
\rho_{f}\left(u \frac{\partial u}{\partial x}+v \frac{\partial u}{\partial y}\right)=-\frac{\partial P}{\partial x}+\frac{\partial}{\partial x}\left(\mu \frac{\partial u}{\partial x}\right)+\frac{\partial}{\partial y}\left(\mu \frac{\partial u}{\partial y}\right)
$$

Y-momentum equation:

$$
\rho_{f}\left(u \frac{\partial v}{\partial x}+v \frac{\partial v}{\partial y}\right)=-\frac{\partial P}{\partial y}+\frac{\partial}{\partial x}\left(\mu \frac{\partial v}{\partial x}\right)+\frac{\partial}{\partial y}\left(\mu \frac{\partial v}{\partial y}\right)+\rho g \beta\left(T-T_{\text {ref }}\right)
$$

Energy equation: 


$$
\rho_{f} C_{p}\left(u \frac{\partial T}{\partial x}+v \frac{\partial T}{\partial y}\right)=\frac{\partial}{\partial x}\left(k_{f} \frac{\partial T}{\partial x}\right)+\frac{\partial}{\partial y}\left(k_{f} \frac{\partial T}{\partial y}\right)
$$

$k$-equation:

$$
\frac{\partial\left(\rho u_{i} k\right)}{\partial x_{i}}=\frac{\partial}{\partial x_{j}}\left(\Gamma_{k} \frac{\partial k}{\partial x_{j}}\right)+\bar{G}_{k}+Y_{k}+S_{k}
$$

where $\bar{G}_{k}, Y_{k}$, and $S_{k}$ stand for turbulent kinetic energy generation, turbulence dissipation, and source term, respectively.

$\omega$-equation:

$$
\frac{\partial\left(\rho u_{i} \omega\right)}{\partial x_{i}}=\frac{\partial}{\partial x_{j}}\left(\Gamma_{\omega} \frac{\partial \omega}{\partial x_{j}}\right)+G_{\omega}-Y_{\omega}+D_{\omega}+S_{\omega}
$$

where $G_{\omega}, Y_{\omega}, D_{\omega}$ and $S_{\omega}$ stand for the specific dissipation rate, $\omega$-dissipation, crossdiffusion, and source term, respectively.

The quadrilateral domain cells are adopted for meshing; the mesh is clustered with five layers near the wall. The receiver is located in a virtual surrounding to estimate heat loss. The boundary conditions are an isothermal boundary, $\mathrm{T}_{\mathrm{w}}=600 \mathrm{~K}$, at the inner wall of the receiver, adiabatic boundary at other walls of the receiver, velocity inlet condition at wind environment inlet wall. As the internal surface of the cavity receiver is maintained at a high wall temperature, the Boussinesq approximation is not appropriate [15]. Due to high surface temperature, the wall starts emitting radiation and heats up the immediate surrounding, but its impact on convective heat loss is insignificant [16]. Therefore, only the convection model is implemented in the present model. The heat loss due to convection can be estimated by taking the area-weighted average of wall heat flux of cavity internal wall. The ambient air is considered as the incompressible ideal gas, and its properties are temperature-dependent [6].

To reduce computational time, two-dimensional modeling is considered in the present study. However, the investigation is performed to compare the 2-D and 3-D models, and deviation is found to be within $8 \%$ for heat loss from the receiver surface. Therefore, the present model is assumed to be reliable for analysis. The governing equations have been discretized using ANSYS Fluent 20R1 with an implicit solver. The coupled scheme and second order are used for pressure-velocity linkage and pressure computation, respectively. The upwind scheme of second order is chosen for momentum, energy, turbulence kinetic energy, and specific dissipation rate. The criteria for convergence are: the sum of residual for continuity and energy equations should not be more than $10^{-4}$ and $10^{-6}$. The grid independence test has been performed using element sizes from 499239 to 820872 for $\mathrm{d} / \mathrm{h}=1$. The deviation in heat loss is less than $1.5 \%$ between the consecutive element sizes, which is the criteria for grid independence. Due to the unavailability of experimental data of this cavity receiver, the present model is validated with data from a numerical investigation [17] and is found the deviation of $\pm 9 \%$ between the models.

\section{Result and discussions}

The heat loss estimation has been done for various influential parameter variations, such as receiver orientation, diameter to height ratio, wind speed in head-on and back-on direction. In this study, the heat losses are manifested as heat flux $\left(\mathrm{W} / \mathrm{m}^{2}\right)$ for the comparison among 
the investigated configurations. Fig. 2(a), (b) and (c) present the velocity contours in forced convection with $4 \mathrm{~m} / \mathrm{s}$ wind in head-on for $\gamma=0^{\circ}, 45^{\circ}$ and $90^{\circ}$. The orientation of the receiver changes from $0^{\circ}$ to $90^{\circ}$, the development of pressure inside the receiver occurs, which may increase the admission of the incoming air. The pressure across the receiver due to air flowing is termed as adverse pressure gradient. The formed adverse pressure gradient with changing the orientation is one of the causes of heat loss. Furthermore, the heat loss depends on wind speed, the volume of incoming and outgoing air, circulation behaviors, and the stagnation zone inside the receiver. Fig. 2(d), (e), and (f) illustrate the temperature contours in $4 \mathrm{~m} / \mathrm{s}$ wind in head-on. The stagnation zone for $0^{\circ}$ is very thin and close to the wall, due to sufficient air circulation and more hot air sweeping from the receiver, the stagnation zone is not developed inside the receiver. Further, it is noticed with change in orientations, as shown in Fig. 2(d), (e), and (f), the stagnation zone escalates, and it achieves a maximum at $90^{\circ}$. Interestingly, Clausing [9] also noticed a similar phenomenon.

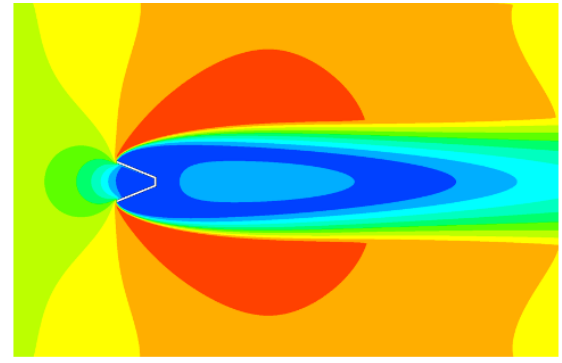

(a)

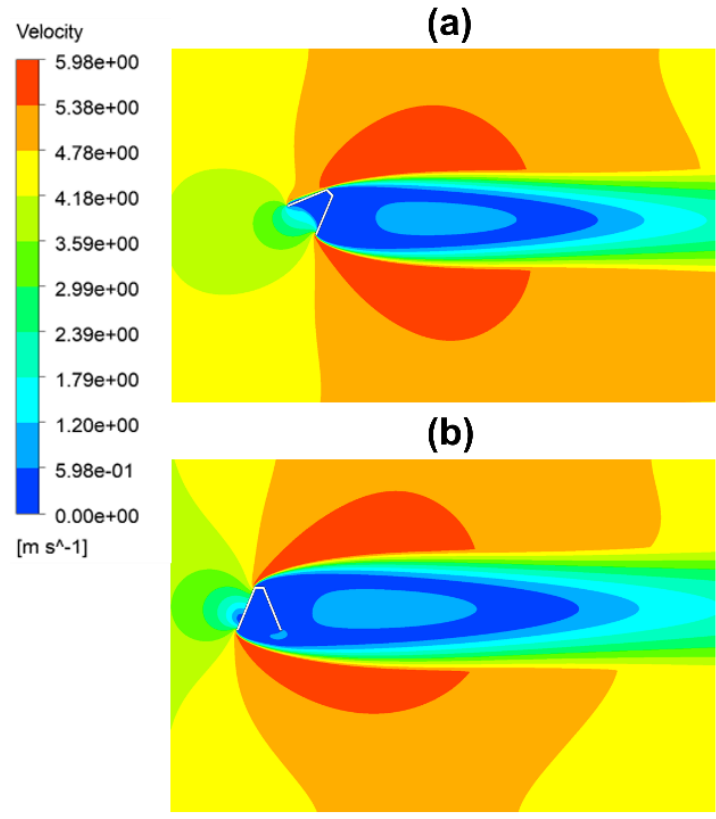

(c)

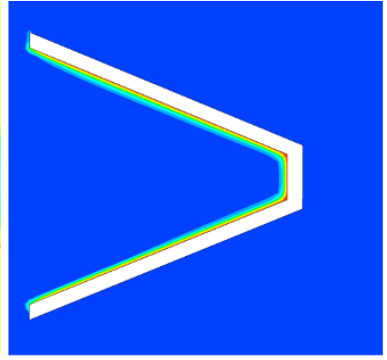

(d)

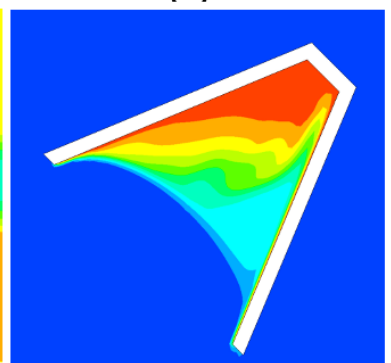

(e)

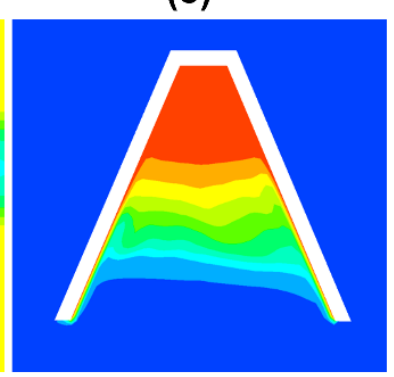

(f)

Fig. 2. Velocity and temperature contours of $d / h=1$ for the head-on wind speed of $4 \mathrm{~m} / \mathrm{s}$ at $0^{\circ}(\mathrm{a}, \mathrm{d})$, $45^{\circ}(\mathrm{b}, \mathrm{e})$, and $90^{\circ}(\mathrm{c}, \mathrm{f})$.

Fig. 3(a), (b) and (c) demonstrate the heat loss with receiver orientation and wind speed in head-on direction for $\mathrm{d} / \mathrm{h}=0.5,1$, and 1.5 , respectively. The receiver orientation, $\gamma=0^{\circ}$ corresponds to altitude angle of $0^{\circ}$ (sunrise condition) and $\gamma=90^{\circ}$ harmonized with altitude 
angle of $90^{\circ}$ (noon condition). For $90^{\circ}$ to $180^{\circ}$ orientations, it can be considered as receiver direction of $0-90^{\circ}$. Therefore, the orientation of $0^{\circ}$ is chosen as limiting value for both $\gamma=0^{\circ}$ and $180^{\circ}$. It is noticed from Fig. 3 that the effect of forced convection is not strong enough at $\mathrm{V} \leq 2 \mathrm{~m} / \mathrm{s}$, the heat loss variation in the windy condition is close or less than that in nowind condition (natural convection). The heat loss variations with orientation in natural convection are within $12 \%$ for all $\mathrm{d} / \mathrm{h}$ values. For high wind speeds, there are established heat loss trends with receiver orientations. The variation in heat loss up to $30^{\circ}$ for $\mathrm{d} / \mathrm{h}=0.5$ is more, and the curves are close to each other than $\mathrm{d} / \mathrm{h}=1$ and 1.5 . However, heat loss magnitude increases with increasing $\mathrm{d} / \mathrm{h}$. Further, with the increase in orientations, heat loss increases and attains the maximum at $60^{\circ}$ and $75^{\circ}$ for $\mathrm{d} / \mathrm{h}=0.5$ and $1-1.5$ respectively, then heat loss decrease to the minimum at $90^{\circ}$ for $\mathrm{V}>2 \mathrm{~m} / \mathrm{s}$. At high heat loss, the convective region dominates, as shown in Fig. 2(e), due to more incoming air and circulation inside the receiver. In contrast, at the lowest heat loss, less air going inside the receiver, stagnation region dominates, as shown in Fig. 2(f).

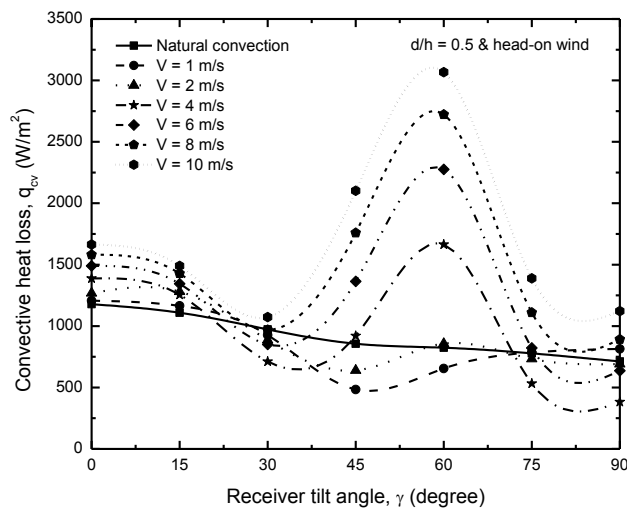

(a)

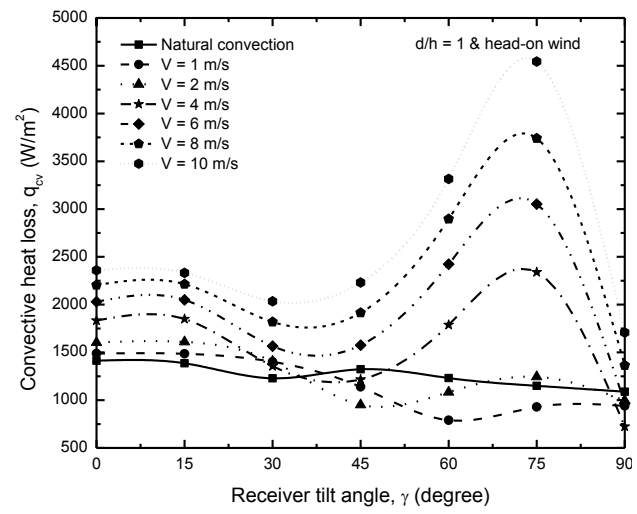

(b)

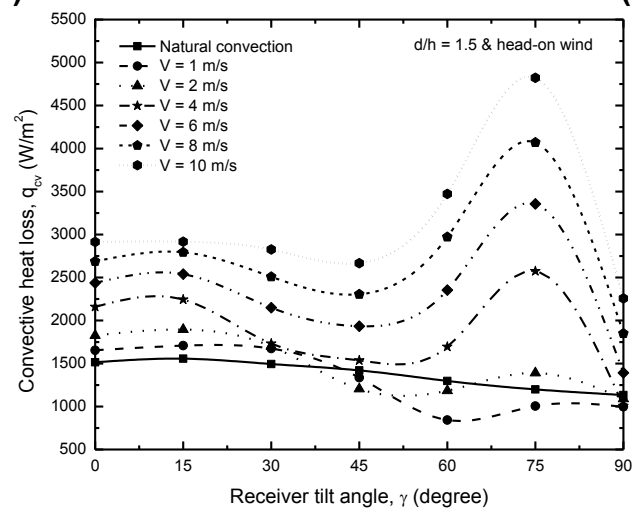

(c)

Fig. 3. Impact on convective heat loss with varying head-on wind speed (a) $d / h=0.5$, (b) $d / h=1$, and (c) $\mathrm{d} / \mathrm{h}=1.5$.

Fig. 4(a), (b) and (c) demonstrate the heat loss variation with orientation and wind speed in back-on direction for $\mathrm{d} / \mathrm{h}=0.5,1$, and 1.5 , respectively. The heat loss patterns for $\mathrm{V} \geq 4$ $\mathrm{m} / \mathrm{s}$ are similar and non-intersecting, but the same is not observed for low wind speed. For low wind speed, the heat loss variation is not high with orientations. The forced convection is not more significant than natural convection at low wind speed for low orientations. For higher orientations, natural convection heat loss dominates more than wind conditions at low 
wind speed and some of higher value; this indicates the heat loss reduces in wind condition owing to repression of hot air, which is about to leave the receiver. The variation in heat loss in no-wind condition with orientation decreases with increasing $\mathrm{d} / \mathrm{h}$ value. The heat loss for all $\mathrm{d} / \mathrm{h}$ values is higher at $30^{\circ}$ in back-on wind direction and further decreases with increasing orientation. This heat loss reduction is due to insufficient wind movement for sweeping hot air, increasing the stagnation zone formed inside the receiver.

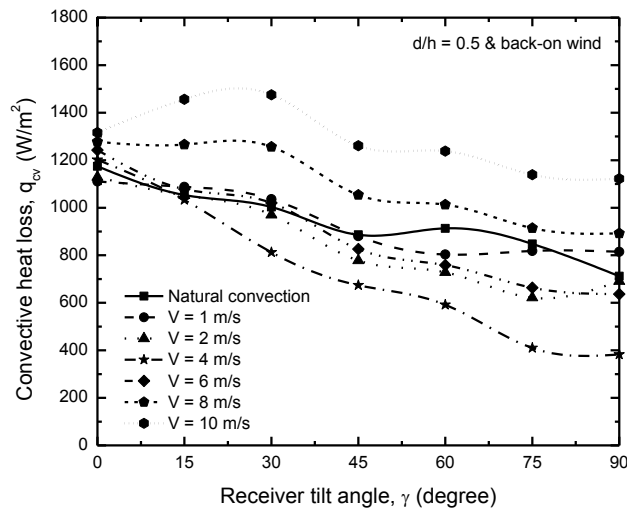

(a)

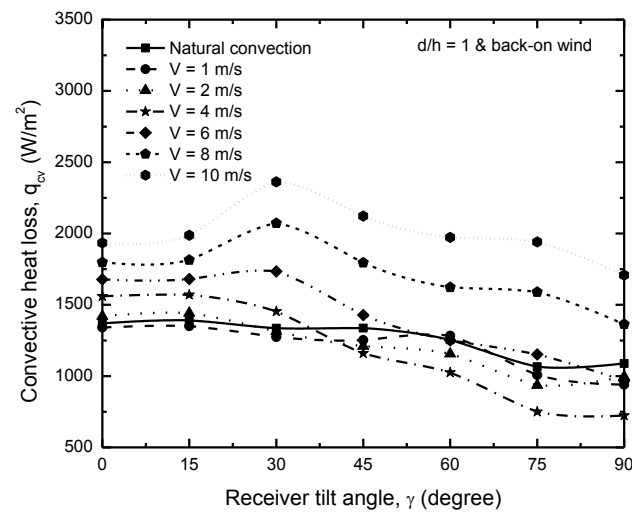

(b)

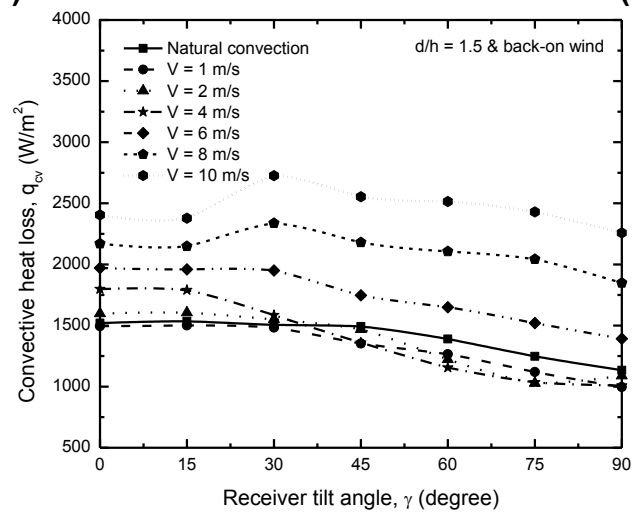

(c)

Fig. 4. Impact on convective heat loss with varying back-on wind speed (a) $d / h=0.5$, (b) $d / h=1$, and (c) $\mathrm{d} / \mathrm{h}=1.5$.

\section{Conclusion}

The numerical investigation was performed to estimate the heat losses due to convection from the cavity receiver with variation in wind speeds, wind conditions, orientations, $d / h$ ratios. The wind effect caused the flow separation and adverse pressure gradient, due to which the receiver was subjected to heat loss. In the current study, the critical value of wind speed has been revealed for operating conditions below which the force convection effect is diminished. The critical wind speed was found to be lower than $4 \mathrm{~m} / \mathrm{s}$, it varies as per orientations, wind conditions, and $\mathrm{d} / \mathrm{h}$ values. The highest heat loss occurred at the orientation of $60^{\circ}$ and $75^{\circ}$ for $\mathrm{d} / \mathrm{h}=0.5$ and $1-1.5$, respectively, for high wind speed in head-on, while in back-on, it occurred at $30^{\circ}$. The lowest heat loss occurred at the orientation of $90^{\circ}$ for $4-10$ $\mathrm{m} / \mathrm{s}$. The present estimation of heat loss can be helpful in the performance evaluation of parabolic dish collector for industrial process heat of medium and high temperatures. 


\section{References}

1. M. Wang and K. Siddiqui, Renew. Energy, 35 (11), 2501 (2010)

2. J. P. Holman, Heat Transfer, McGraw-Hill (1992)

3. T.L.Bergman, L. A.S, I. F.P., and D. P. DeWitt, Fundamentals of Heat and Mass Transfer, 7th edition. JOHN WILEY \& SONS SEVENTH (2011)

4. L. Xiao, S. Y. Wu, and Y. R. Li, Int. J. Therm. Sci., 60, 182 (2012)

5. S. Paitoonsurikarn, T. Taumoefolau, and K. Lovegrove, Solar, 1 (2004)

6. K. S. Reddy, G. Veershetty, and T. Srihari Vikram, Sol. Energy, 131, 183 (2016)

7. T. S. Vikram and K. S. Reddy, Int. J. Therm. Sci., 87, 19 ( 2015)

8. K. S. Reddy, T. S. Vikram, and G. Veershetty, Sol. Energy, 121, 78 (2015)

9. A. M. Clausing, Sol. Energy, 27(4), 295 (1981)

10. T. Taumoefolau, S. Paitoonsurikarn, G. Hughes, and K. Lovegrove, Journal of Solar Energy Engineering, Transactions of the ASME, 126(2), 801 (2004)

11. U. Leibfried and J. Ortjohann, J. Sol. Energy Eng. Trans. ASME, 117(2), 75 (1995)

12. R. Flesch, H. Stadler, R. Uhlig, and B. Hoffschmidt, Appl. Therm. Eng., 87, 724 (2015)

13. M. Prakash, S. B. Kedare, and J. K. Nayak, Int. J. Therm. Sci., 49(4), 680 (2010)

14. E. Abbasi-Shavazi, G. O. Hughes, and J. D. Pye, Energy Procedia, 69, 269 (2015)

15. K. S. Reddy, S. Balaji, and T. Sundararajan, Energy, 150, 410(2018)

16. S. Paitoonsurikarn and K. Lovegrove, Proc. 44th Annu. Conf. Aust. New Zeal. Sol. Energy Soc. (2006)

17. M. Uzair, T. N. Anderson, and R. J. Nates, Sol. Energy, 176, 496 (2018) 